\title{
DIFFEOMORPHISMS OF MANIFOLDS AND SEMIFREE ACTIONS ON HOMOTOPY SPHERES
}

\author{
BY WILLIAM BROWDER AND TED PETRIE \\ Communicated by M. H. Protter, June 18, 1970
}

The paper of which this is an announcement is naturally divided into two parts. One is the study of the group of pseudoisotopy classes of diffeomorphisms of a particular class of manifolds. The second is the use of the results of this study to construct and classify the "group" of semifree $H$ actions on homotopy $2 k+q+1$ spheres with a homotopy $q$ sphere as fixed point set. Here $H$ is a compact Lie Group and a semifree $H$ action on a manifold $M$ is a smooth action on $M$ such that the action is free off the fixed point set of $H$. The class of groups which can act semifreely on any manifold is restrictive but rich and this class has been determined. It coincides with the set of groups which have a real representation such that no element of the group other than the identity has one as an eigenvalue. Equivalently, it is the set of groups which can act freely and linearly on a standard $k$-sphere $S^{k}$.

Particularly interesting consequences of our study are these: If $H$ acts freely on $S^{k}$, let $P^{k}=S^{k} / H$ be the orbit space and $D\left(P^{k, q}, \partial\right)$ be the group of pseudoisotopy classes rel boundary of diffeomorphisms of $P^{k} \times D^{q}$ which are the identity on $P^{k} \times S^{q-1}$. If $H$ is $Z_{p}, p$ odd, $S^{0}$, $S^{1}, S^{3}$ or $N$ the normalizer of $S^{1}$ in $S^{3}$, we can give a complete description of the torsion free part of $D\left(P^{k, q}, \partial\right)$. For other groups $H$, if $k$ is sufficiently large compared with $q$, we can determine this group modulo knowledge of the Wall surgery obstruction group

$$
L_{n}^{s}\left(\pi_{0}(H), \omega(H)\right), \quad n=k+q-\operatorname{dim} H+1 .
$$

Let $a^{k, q}=$ the set of isomorphism classes of semifree $H$ actions on homotopy $(k+q+1)$-spheres with a homotopy $q$-sphere as fixed point set. We show that a certain subset $a_{0}^{k, q} \subset a^{k, q}$ has the natural structure of an abelian group. We exhibit an epimorphism of groups $D\left(P^{k, q}, \partial\right) \rightarrow Q_{0}^{k, q}$ and determine the torsion free part of the group $a_{0}^{k . q}$ in certain cases. In particular, we settle a question of [1] showing that there are only a finite number of inequivalent semifree $S^{1}$

AMS 1969 subject classifications. Primary 5720, 5747.

Key words and phrases. Surgery, homotopy theory, smooth actions.

Copyright (c) 1971. American Mathematical Society 
actions on homotopy spheres with an even dimensional homotopy sphere as fixed point set.

Here is the motivation for this work. Let $g$ be an $H$ equivariant diffeomorphism of $S^{k} \times D^{q}$ - which is the identity on $S^{k} \times S^{q-1}$. Extend $g$ to $S^{k} \times S^{q} \supset S^{k} \times D_{-}^{q}$ by defining $g \mid S^{k} \times D_{+}^{q}$ to be the identity. Here $D_{+}^{q}$ and $D_{-}^{q}$ are the upper and lower hemispheres in $S^{q}$. The manifold $\Sigma(g)=D^{k+1} \times S^{q} \cup_{g} S^{k} \times D^{q+1}$ obtained by gluing along $S^{k} \times S^{q}$ with $g$ is a homotopy sphere supporting a semifree $H$ action defined by $h(x, y)=(\rho(h) x, y)$ when $x \in D^{k+1}, y \in D^{q+1}$ and either $\|x\|=1$ or $\|y\|=1$ and $\rho: H \rightarrow S O_{k+1}$ is a fixed point free representation of $H$. This action has these properties:

(P) The fixed point set is the standard sphere $S^{q}$. The normal bundle is trivial and the action of $H$ on the normal fibers is defined by $\rho$.

For any semifree $H$ action on a homotopy $k+q+1$ sphere, a torsion is defined as an element of the Whitehead group of $\pi_{0}(H)=\pi$ (see [6]). The set of those actions satisfying (P) and having torsion zero is the group $Q_{0}^{k, q}(\rho)$.

THEOREM 1. The correspondence $g \rightarrow \Sigma(g)$ is a well-defined epimorphism of abelian groups $D\left(P^{k, q}, \partial\right) \stackrel{\mu}{\rightarrow} a_{0}^{k, q}(\rho)$.

The kernel of $\mu$ depends on $\rho$. Specifically let $C$ be the centralizer of $\rho(H)$ in $S O_{k+1}$. There is a homomorphism $\pi_{q}(C) \rightarrow D\left(P^{k, q}, \partial\right)$.

Theorem 2. $\pi_{q}(C) \rightarrow D\left(P^{k, q}, \partial\right) \rightarrow \mathfrak{Q}_{0}^{k, q}(\rho) \rightarrow 0$ is exact.

The techniques for studying the group $D\left(P^{k, q}, \partial\right)$ are surgery and homotopy theory. We describe two exact sequences:

$$
\begin{aligned}
\text { (S) } \cdots & \stackrel{\sigma}{\rightarrow} L_{n+q+1}(\pi) \stackrel{\omega}{\rightarrow} h \mathfrak{S}\left(P^{k} \times D^{q}, P^{k} \times S^{q-1}\right) \\
& \stackrel{d}{\rightarrow}\left[P^{k} \times D^{q}, P^{k} \times S^{q-1} ; G / 0\right] \stackrel{\sigma}{\rightarrow} L_{n+q}(\pi) \rightarrow \cdots \\
& n=k-\operatorname{dim} B=\operatorname{dim} P^{k}, \text { and } \pi=\pi_{1}\left(P^{k}\right) . \\
\text { (D) } \cdots & \rightarrow \pi_{q+1}\left(G P^{k}\right) \stackrel{\psi}{\rightarrow} h s\left(P^{k} \times D^{q+1}, P^{k} \times S^{q}\right) \\
& \stackrel{\partial}{\rightarrow} D\left(P^{k, q}, \partial\right) \stackrel{\lambda}{\rightarrow} \pi_{q}\left(G P^{k}\right) \rightarrow \cdots
\end{aligned}
$$

which provide a very good description of $D\left(P^{k, q}, \partial\right)$. The sequence (S) is the usual surgery exact sequence discussed for example in [4], [5]. The space $G P^{k}$ appearing in (D) is the identity component of the space of maps of $P^{k}$ to itself.

We use the homotopy and surgery theory of (S) and (D) to obtain 
$D\left(P^{k, q}, \partial\right)$ in terms of the Wall groups $L_{n}(\pi)$. Happily there has been a pleasant interplay between the geometry and algebra which has allowed one of the authors [2], [3] to obtain algebraic results on $L_{n}(\pi)$ from the geometry. Now that these groups are falling to the algebraic attacks, we should soon have complete information on $L_{n}(\pi)$ for our description of semifree actions.

Here are some of the more important results which can be simply stated: Let $p$ be an odd prime and $\rho: Z_{p} \rightarrow U((k+1) / 2)$ a unitary fixed point free representation of complex dimension $(k+1) / 2$. Then $\rho=\sum_{j=1}^{p-1} n_{j} \gamma^{j}$ where $\gamma$ is the basic complex one dimensional representation of $Z_{p}$ defined by multiplication by $\exp 2 \pi i / p$. Set $n(\rho)$ $=\max \left(2 n_{i}-1\right)$.

THEOREM 3. In Theorem 2, the image of $\pi_{q}(C)$ in $D\left(P^{k, q}, \partial\right)$ is finite if $n(\rho)<q$. If $n(\rho) \geqq q$, then the rank of the image is $\lambda(\rho)$ if $q$ is odd and 0 if $q$ is even, where $0 \leqq \lambda(\rho) \leqq(p+1) / 2$.

TheOREM 4. Under the above hypotheses,

$$
D\left(P^{k, q}, \partial\right) \otimes \mathfrak{C}=V_{k+q+2}\left(Z_{p}\right)+\bigwedge_{0}^{k, q+1}
$$

where, for $m$ even, $V_{m}\left(Z_{p}\right)=$ the group of complex valued functions on the nontrivial pth roots of unity satisfying $f\left(t^{j}\right)=(-1)^{m / 2} f\left(t^{p-j}\right), t$ $=\exp (2 \pi i / p), V_{m}\left(Z_{p}\right)=0$ for $m$ odd,$\Lambda^{k, q+1}=\mathfrak{C}$ for $q+1 \equiv 0(4)$, and $\Lambda^{k, q+1}=0$ otherwise.

The group $\mathfrak{Q}_{0}^{k, q}(\rho) \otimes \mathcal{C}$ can now be described as the cokernel of a map $\Delta \oplus \psi: \pi_{q}(C) \otimes \mathfrak{e} \rightarrow V_{k+q+2}\left(Z_{p}\right)+\Lambda_{0}^{k, q+1}$. Regarding an element of $\pi_{q}(C)$ as a complex bundle over $S^{q+1}$, we can describe the map $\psi$ simply in terms of the Chern classes of this bundle. The map $\Delta$ also has an explicit description in terms of these Chern classes, using the Atiyah-Singer Index Theorem, but this description is more complicated.

Let $N$ be the normalizer of $S^{1}$ in $S^{3}$.

THEOREM 5. For the groups $S^{1}, N$ and $S^{3}$ there is essentially one fixed point free representation possible on the normal fibers to the fixed point set. In this case we have $a_{0}^{k, q} \otimes Q \cong \Lambda_{0}^{k, q+1}$

where

$$
\Lambda_{0}^{k, q+1} \subset H^{4 *}\left(P^{k} \times\left(D^{q+1}, S^{q}\right) ; Q\right)
$$

is a subspace of codimension 1 if $n+q+1 \equiv 0$ (4) and of codimension 0 if $n+q+1 \not \equiv 0$ (4) where $n=k-\operatorname{dim} H$. 
TheOREM 6. For the group $Z_{2}=S^{0}$, we have

$$
a_{0}^{k, q} \otimes Q \cong B_{k, q}+\dot{L}_{k+q+2}^{0}\left(Z_{2},(-1)^{k+1}\right) \otimes Q
$$

where $L_{i}^{0}\left(Z_{2},(-1)^{k+1}\right) \otimes Q=0$ unless $(k+1)$ is even and $i \equiv 0$ (4) and $L_{4 k}^{0}\left(Z_{2},+1\right) \otimes Q, B_{k, q}=0$ unless $q \equiv-1$ (4) and $q \geqq 2 k+1$ in which case $B_{k, q}=Q$.

\section{REFERENCES}

1. W. Browder, Surgery and the theory of differentiable transformation groups, Proc. Conference on Transformation Groups (New Orleans, 1967) Springer-Verlag, New York, 1969.

2. T. Petrie, The Atiyah-Singer invariant, the Wall groups $L_{n}(\pi, 1)$ and the function $t e^{x} 1 / t e^{x}-1$, Ann. of Math. 92 (1970), 174-187.

3. - Surgery with coefficients in a field (to appear).

4. D. Sullivan, Triangulating and smoothing homotopy equivalences and homeomorphisms, Princeton University, Princeton, N. J., 1967. (mimeographed notes)

5. C. T. C. Wall, Surgery on compact manifolds (to appear).

6. M. Rothenberg and J. Sondow, Non-linear smooth representations of compact Lie groups (to appear).

Princeton University, Princeton, New Jersey 08540 